\title{
Criminologie
}

\section{Intimidation en milieu carcéral : effet sur les pratiques professionnelles des agents correctionnels du Québec}

\section{Grégory Gomez del Prado}

Volume 45, numéro 2, automne 2012

URI : https://id.erudit.org/iderudit/1013729ar

DOI : https://doi.org/10.7202/1013729ar

Aller au sommaire du numéro

\section{Éditeur(s)}

Les Presses de l’Université de Montréal

ISSN

0316-0041 (imprimé)

1492-1367 (numérique)

Découvrir la revue

Citer cet article

Gomez del Prado, G. (2012). Intimidation en milieu carcéral : effet sur les pratiques professionnelles des agents correctionnels du Québec. Criminologie, 45(2), 301-322. https://doi.org/10.7202/1013729ar
Résumé de l'article

Les études portant sur la victimisation des surveillants de prison ont souligné la relative rareté des agressions sévères commises par les détenus. L’univers carcéral est plutôt ponctué de violences physiques mineures et d'attaques psychologiques contre les gardiens. Si plusieurs recherches ont mis en évidence les facteurs d'influence des pratiques professionnelles des gardiens de prison, peu d'études se sont penchées sur l'impact de la violence sur leur travail. Un questionnaire structuré a été soumis à 132 agents correctionnels travaillant dans des établissements de juridiction provinciale dans la région de Montréal afin d'établir les niveaux et les formes d'intimidation vécue par ces agents et afin d'évaluer les effets de cette victimisation sur leur travail. D'une part, nos analyses confirment les tendances générales de victimisation des gardiens de prison. D'autre part, cette victimisation semble modifier certaines pratiques professionnelles. D'autres études sont toutefois nécessaires afin de valider le concept de pratiques professionnelles en milieu carcéral. 


\title{
Intimidation en milieu carcéral: effet sur les pratiques professionnelles des agents correctionnels du Québec
}

\author{
Grégory Gomez del Prado \\ Candidat au doctorat \\ École de criminologie, Université de Montréal \\ ggomezdelprado@gmail.com
}

\begin{abstract}
RÉSUMÉ • Les études portant sur la victimisation des surveillants de prison ont souligné la relative rareté des agressions sévères commises par les détenus. L'univers carcéral est plutôt ponctué de violences physiques mineures et d'attaques psychologiques contre les gardiens. Si plusieurs recherches ont mis en évidence les facteurs d'influence des pratiques professionnelles des gardiens de prison, peu d'études se sont penchées sur l'impact de la violence sur leur travail. Un questionnaire structuré a été soumis à 132 agents correctionnels travaillant dans des établissements de juridiction provinciale dans la région de Montréal afin d'établir les niveaux et les formes d'intimidation vécue par ces agents et afin d'évaluer les effets de cette victimisation sur leur travail. D'une part, nos analyses confirment les tendances générales de victimisation des gardiens de prison. D'autre part, cette victimisation semble modifier certaines pratiques professionnelles. D'autres études sont toutefois nécessaires afin de valider le concept de pratiques professionnelles en milieu carcéral.
\end{abstract}

MOTS-CLÉs - Violence en milieu de travail, intimidation criminelle, gardiens de prison, victimisation, pratiques professionnelles.

\section{Introduction $^{1}$}

À l'instar des policiers, les gardiens de prison ${ }^{2}$ sont des dépositaires de l'autorité publique dont la principale caractéristique est le recours

1. L'auteur tient à remercier Laura Benghozi et Marion Desfachelles pour leur collaboration lors de la collecte des données ainsi que le personnel des Services correctionnels du Québec (SCQ) pour leur aimable participation. L'auteur remercie également Étienne Blais et Maurice Cusson pour leurs commentaires à la fois pertinents et constructifs dans la rédaction de cet article. Les analyses présentées dans cet article n'engagent que l'auteur et ne sont pas nécessairement représentatives des opinions du SCQ.

2. Dans cet article, les termes gardien, surveillant et agent correctionnel ont la même signification et seront donc utilisés de manière indiscriminée.

Criminologie, vol. 45, n 2 (2012) 
légitime à la force physique (Bittner, 1990). Dans un contexte de contacts répétés et inévitables avec les détenus, le rapport de force ne s'établit toutefois pas de la même manière. Si l'aspect coercitif est indissociable des tâches du gardien de prison, il apparait que ses rapports avec les délinquants sont marqués par la négociation et la réciprocité (Lombardo, 1981; Sykes, 2007). L'utilisation de la force comme unique moyen d'assurer l'ordre étant nettement insuffisante, le calme en prison est négocié sur la base d'échanges et de services mutuels (Benguigui, 1997; Chantraine, 2004).

La négociation permet donc aux deux parties d'éviter les conflits perpétuels et d'instaurer un certain ordre à l'intérieur des murs. Ce «professionnalisme informel» (Benguigui et al., 1992) doit cependant être teinté de la menace de la sanction. Pour arriver à maintenir l'ordre, les surveillants doivent doser judicieusement leur niveau de coercition en sachant sanctionner au moment opportun. Dans un milieu où cohabitent des individus ayant un potentiel élevé de violence et dont la caractéristique commune est d'avoir enfreint la loi, l'application de règles et de sanctions peut engendrer des réactions agressives chez des détenus. Confrontés, pour certains, à des spécialistes de l'intimidation, les rapports peuvent alors prendre la forme de menaces ou d'agressions physiques contre les membres du personnel.

Les études portant sur la victimisation des surveillants de prison ont souligné la relative rareté des agressions sévères commises par les détenus (Light, 1991; Patrick, 1998; Ouimet, 1999; Jiang et Fisher-Giorlando, 2002; Useem et Piehl, 2006; Lahm, 2009; Sorensen et al., 2011). Ainsi, les gardiens de prison se situent dans une catégorie professionnelle moins à risque que les huissiers ou les policiers (Lusignan, 1995). Nonobstant, plusieurs auteurs ont mis en lumière l'omniprésence d'un sentiment d'insécurité au sein de la profession carcérale (Jacobs et Retsky, 1975; Kauffman, 1988; Robinson et al., 1997; Adler, 1998; Vacheret et Milton, 2007). Alors que les conséquences de cette insécurité, du stress ou encore du conflit des rôles chez les gardiens de prison ont largement été abordées (Hepburn et Albonetti, 1980; Poole et Regoli, 1980; Cheek et Miller, 1983; Triplett et al., 1996; Ben-David et al., 1996; Schaufeli et Peeters, 2000; Griffin, 2002), peu d'études se sont intéressées à l'impact de la victimisation des agents correctionnels.

Dans cet article, nous nous penchons sur les répercussions d'une forme particulière de violence vécue par les gardiens de prison: les actes ou les tentatives d'intimidation perpétrés par les détenus. Nous avançons 
l'hypothèse que les intimidations répétées, exprimées à travers des menaces ou des actes physiques mineurs, auront un impact sur le travail quotidien des gardiens de prison. Cette question est particulièrement pertinente car les surveillants évoluent dans un milieu où le niveau de répression et de tolérance nécessaire est constamment soumis à leur appréciation personnelle. Dès lors, il est envisageable que des pressions psychologiques influencent indûment leur jugement et modifient leur façon d'intervenir auprès des détenus.

\section{Recension des écrits}

\section{La victimisation des gardiens de prison}

Indépendamment de la méthodologie utilisée, les recherches portant sur la victimisation des gardiens de prison soulignent la relative rareté et la faible gravité des agressions perpétrées par les détenus. Dans une analyse de plus de 600 rapports d'incidents des prisons de l'État de New York, Light (1991) a constaté que plus de $80 \%$ des agressions contre les gardiens n'avait entraîné que des blessures physiques mineures, voire aucune blessure. Entre 1995 et 2000, Stephan et Karberg (2003) ont remarqué que le nombre d'agressions mortelles avait baissé dans l'ensemble des établissements de détention aux États-Unis malgré une hausse du nombre de détenus et de membres du personnel. Ainsi, le taux de violence contre les employés des prisons américaines se situerait aux alentours de 15 agressions par 1000 détenus. Confirmant ces résultats sur une période d'environ 30 ans, Useem et Piehl (2006) ont également remarqué que le nombre d'émeutes et de meurtres de gardiens de prison avait grandement diminué depuis les années 1970. Kratcoski (1988) a souligné le caractère à la fois mineur et spontané des agressions commises par les incarcérés. Dans une étude plus récente, Lahm (2009) a constaté que seulement $4 \%$ des 1054 détenus, issus d'une trentaine de prisons aux États-Unis, avaient commis des agressions contre leurs surveillants. De leur côté, Sorensen et al. (2011) ont établi le taux de violence aggravée contre le personnel à 2,84 agressions par 1000 employés. Finalement, dans le but d'établir un portrait de la situation dans les établissements de juridiction provinciale au Québec, Ouimet (1999) a constaté que, sur plus de 8500 rapports d'infractions, moins de $2 \%$ des dossiers impliquaient de la violence commise par les détenus à l'endroit des surveillants. 
Même si les agressions sévères sont rares, les violences psychologiques et les attaques personnelles semblent ponctuer le quotidien des surveillants (Jiang et Fisher-Giorlando, 2002; Lahm, 2009). Dans une étude menée auprès d'agents correctionnels fédéraux, Vacheret et Milton (2007) ont constaté que les trois quarts des sujets interrogés avaient été victimes de dénigrements et d'insultes de la part des détenus et que la moitié d'entre eux se disaient victimes de menaces. Par ailleurs, les auteurs ont souligné l'omniprésence d'un sentiment d'insécurité au sein de la profession pénitentiaire qui s'amplifiait lorsque le surveillant était appelé à exercer des tâches coercitives. Ces craintes ne seraient cependant pas reliées à des expériences réelles de victimisation. Dans une étude menée en Angleterre et au Pays de Galles, Adler (1998) a noté que les deux tiers des sujets exprimaient des peurs et des craintes, notamment par rapport aux confrontations avec les détenus et à une éventuelle perte de contrôle de la situation. Des résultats semblables ont été constatés par Robinson et al. (1997) au Canada : plus des trois quarts des gardiens dans les pénitenciers fédéraux estimaient que les détenus pouvaient mettre en danger leur sécurité personnelle.

\section{Facteurs d'influence des pratiques professionnelles}

Si l'univers carcéral est composé de peurs et d'incertitudes, trois réponses adaptatives ont été observées chez les gardiens: ils devenaient encore plus répressifs; ils cherchaient à être acceptés par les détenus; ou ils fuyaient leurs responsabilités professionnelles (Jacobs et Retsky, 1975). Dans une recherche effectuée auprès de surveillants d'un établissement à sécurité maximale en Israël, Ben-David et al. (1996) ont constaté que les sujets qui ressentent le plus de craintes par rapport à leur intégrité physique sont les plus punitifs envers les incarcérés. Griffin (2002) a remarqué que les gardiens qui craignent la victimisation définissent davantage leur profession de manière coercitive et punitive. Il apparaît également que plus les gardiens menacent d'utiliser la force physique plus les détenus risquent de réagir violemment (Patrick, 1998). À l'instar des policiers (Corrigan et al., 1980), les surveillants de prison qui se sentent plus en danger ont tendance à être plus autoritaires et plus enclins à utiliser la force physique dans leurs interventions. Ces résultats ont également été soulignés dans les travaux de Vacheret et Milton (2007) qui ont argué que les agents qui ressentent de l'insécurité privilégient un rôle exclusif de surveillance et de contrôle. Plus encore, ces agents 
ont davantage tendance à adopter des stratégies protectrices telles que le retrait défensif, l'évitement ou l'affrontement que leurs collègues qui se sentaient en sécurité. Parallèlement, Lusignan (1999) a observé que la victimisation chez les criminologues cliniciens travaillant auprès de détenus fédéraux modifiait leur pratique professionnelle.

Cette orientation disciplinaire a également été observée au sein des surveillants confrontés aux ambiguiités de leur fonction. Coincés entre un mandat de contrôle et de surveillance, exigeant une attitude ferme, et une mission de réinsertion sociale, demandant de l'écoute et de l'empathie, les gardiens vivent un conflit des rôles (Vacheret et Lemire, 2007). Face à cette contradiction, les surveillants vont accentuer leur rôle répressif parce qu'il permet une évaluation objective de leur performance. En effet, les gardiens connaissent les critères qui définissent leur mandat de contrôle et de surveillance - qu'il n'y ait pas d'altercations avec les détenus et qu'ils fassent un usage minimal de la force alors que les critères d'évaluation du mandat d'entraide sont beaucoup plus opaques (Hepburn et Albonetti, 1980). Pour Poole et Regoli (1980), les gardiens font face au conflit des rôles en privilégiant une approche plus disciplinaire qui les pousse à percevoir et à évaluer différemment les comportements des détenus et, de manière ultime, à être plus sévères avec eux. À l'instar du sentiment d'insécurité, le conflit des rôles amènerait les surveillants à privilégier l'une ou l'autre des trois attitudes suivantes: un renforcement du rôle coercitif; l'accentuation de la mission de réinsertion sociale; ou un retrait et un laisser-aller dans l'exécution des tâches (Vacheret, 2001).

\section{La présente étude}

Il apparaît donc que les violences physiques perpétrées contre les gardiens de prison se limitent souvent à des actes de gravité moindre ou à des violences psychologiques. En marge de cette violence, les gardiens ressentent une forte insécurité dans l'accomplissement de leurs tâches quotidiennes. Alors que les conséquences de cette insécurité ont été largement abordées, l'impact des expériences de victimisation réelle vécues par les gardiens demeure un phénomène méconnu. Au Canada, Rosine (1992) s'est penché sur l'impact psychologique d'un ensemble diffus d'événements traumatisants allant du suicide d'un détenu à l'émeute, en passant par les agressions entre détenus. Seidman et Williams (1999) se sont intéressés aux répercussions des prises d'otage et des séquestra- 
tions vécues par les agents correctionnels fédéraux. Néanmoins, ces incidents graves demeurent exceptionnels et ne sont pas représentatifs des interactions qui rythment le quotidien des surveillants de prison. Plus encore, les études réalisées jusqu'à présent avaient comme objectif de décrire ces situations plutôt que d'en expliquer les répercussions sur les pratiques professionnelles carcérales (Rosine, 1992; Lusignan, 1999; Seidman et Williams, 1999; Vacheret et Milton, 2007). Pourtant, l'étude des changements dans les pratiques des gardiens de prison est centrale afin de mieux comprendre les implications possibles pour leur sécurité et celle des détenus.

Ne possédant pas de légitimité de facto, les surveillants construisent leur autorité de manière individuelle en appliquant avec discernement et discrétion les règlements (Lombardo, 1981; Cooley, 1995). Néanmoins, cette autorité doit donc toujours s'accompagner du spectre de la sanction (Hepburn, 1985). Dans de telles conditions, les intimidations physiques et verbales pourraient remettre en question le pouvoir coercitif des gardiens de prison. La peur étant génératrice de plusieurs comportements, dont la fuite et le combat, un surveillant victime d'intimidation pourrait fermer les yeux pour ne pas avoir de problème ou bien devenir plus rigide et autoritaire avec les détenus. La question de l'intimidation perpétrée par les détenus est donc particulièrement pertinente. D'une part, il est essentiel de maintenir une pression dissuasive constante sur les délinquants pour assurer l'équilibre des forces en prison. D'autre part, une trop grande rigidité ou une trop grande permissivité de la part des surveillants accentuerait la propension à la violence des détenus.

Le premier objectif de notre étude est de déterminer le niveau de victimisation des gardiens de prison. Contrairement à la plupart des recherches (Light, 1991; Useem et Piehl, 2006; Sorensen et al., 2011), notre étude ne se limite pas aux expériences de victimisation directe. Comme le souligne Agnew (2002), les expériences de victimisation vicariante peuvent avoir un impact tout aussi important que les victimisations directes. Ainsi, les personnes qui sont témoins d'une agression ou qui en entendent parler peuvent raisonnablement croire qu'elles pourraient subir cette même victimisation dans un avenir rapproché (Lin et al., 2011). Parallèlement, la période temporelle de la victimisation a également son importance. Si, à l'instar de Lahm (2009), nous nous intéressons aux actes de violence des douze derniers mois, nous estimons que les victimisations tout au long de la carrière des gardiens de prison doivent être prises en considération, notamment pour apprécier l'effet cumulatif de l'intimidation. 
Le deuxième objectif de notre étude est d'évaluer les effets de ces victimisations sur les pratiques professionnelles, notamment pour déterminer si elles engendrent la passivité chez les surveillants. La particularité de notre étude réside dans le fait qu'elle s'intéresse aux surveillants des établissements de juridiction provinciale, une catégorie professionnelle souvent délaissée par les chercheurs (Ouimet, 1999; Griffin, 2002). À l'opposé de leurs collègues fédéraux, les gardiens des prisons provinciales font affaire avec une population carcérale plus transitoire puisqu'elle est composée soit de détenus condamnés à purger des peines de moins de deux ans, soit de prévenus qui sont en attente de leur jugement ${ }^{3}$.

\section{Méthodologie}

Sources et procédure de collecte de données

Durant le mois de mai 2011, nous avons soumis des questionnaires structurés à 132 agents correctionnels travaillant dans deux établissements de détention de juridiction provinciale (l'établissement de détention de Montréal et celui de Rivière-des-Prairies) ainsi qu'au quartier cellulaire du palais de justice de Montréal. Pour le recrutement, nous avons obtenu l'aide d'un responsable sur place qui s'est chargé d'établir une liste des surveillants intéressés à participer à la recherche. Nous avons également profité de notre présence sur les lieux afin de recruter d'autres volontaires ne faisant pas partie de cette liste. Aucune variable telle que l'âge, le sexe ou le fait d'avoir été intimidé, n'a été utilisée dans notre sélection des participants.

La grande majorité des entretiens a été menée de manière individuelle par un membre de l'équipe de recherche sur le lieu de travail des répondants. Exceptionnellement, des entretiens ont eu lieu en groupe pour des raisons pratiques (impossibilité de trouver un local et présence simultanée de plusieurs agents). La durée moyenne des entretiens était de quinze minutes. Le questionnaire a été construit à partir d'un sondage utilisé dans une étude sur les policiers victimes d'intimidation

3. Au Canada, il existe deux types d'établissement carcéral: 1) les prisons, sous juridiction des provinces, pour les détenus condamnés à une sentence d'emprisonnement maximale de moins de 2 ans et pour les prévenus en attente de leur jugement et 2) les pénitenciers, sous juridiction fédérale, pour les détenus condamnés à une sentence d'emprisonnement de plus de 2 ans. 
(Cusson et al., 2011: 16). Nous avons repris la même définition de l'intimidation, c'est-à-dire «des pressions destinées à dissuader une personne de faire son devoir ou de faire valoir ses droits».

\section{Opérationnalisation des variables}

Les variables indépendantes

Victimisation au cours de la carrière. Afin de mesurer ce concept, deux variables ont été créées. La première variable permet de mesurer les expériences directes d'intimidation. Ainsi, nous avons demandé aux répondants s'ils avaient vécu des actes ou des tentatives d'intimidation et d'estimer la fréquence de ces actes selon une échelle de type Likert à cinq propositions : 1) jamais ; 2) rarement ; 3) occasionnellement ; 4) souvent; et 5) très souvent. La deuxième variable permet de mesurer les expériences vicariantes d'intimidation. Ainsi, nous avons demandé aux répondants s'ils avaient été témoins ou s'ils avaient entendu parler de collègues qui étaient victimes d'intimidation et nous leur avons demandé d'estimer la fréquence en utilisant la même échelle de mesure.

Victimisation dans la dernière année. Pour commencer, nous avons demandé aux répondants s'ils avaient été victimes d'intimidation dans les douze derniers mois (échelle de mesure dichotomique oui/non). Dans l'affirmative, nous leur proposions ensuite d'estimer à quelle fréquence ils avaient été victimes de onze actes d'intimidation.

Ces informations ont permis de créer deux variables. La première variable a mesuré la fréquence de l'ensemble des actes d'intimidation dont ont été victimes les gardiens de prison dans la dernière année. Des analyses descriptives préliminaires des onze actes d'intimidation en question ont révélé une forte asymétrie positive pour chaque indicateur. D'autres analyses ont indiqué une piètre consistance interne $(\alpha=0,30)$ ne permettant pas l'addition des onze indicateurs (Nunnally et Bernstein, 1994). Par conséquent, tous les indicateurs ont été transformés en leur base logarithmique naturelle avant d'être additionnés. La valeur 1 fut ajoutée à chaque indicateur étant donné qu'une forte proportion d'entre eux avait une valeur de 0 . L'addition de ces indicateurs transformés a permis d'obtenir une échelle dont la consistance interne était satisfaisante $(\alpha=0,70)$. La deuxième variable a mesuré la diversité des actes d'intimidation dont ont été victimes les gardiens de prison dans la 
dernière année. Cette échelle a été constituée par l'addition des onze indicateurs considérés sous une forme dichotomique $(\alpha=0,75)$. Une transformation logarithmique a été appliquée par la suite en raison d'une forte asymétrie positive.

\section{Les variables dépendantes}

Pour mesurer le concept des pratiques professionnelles, quatre dimensions importantes ont été identifiées dans la littérature puis mesurées à l'aide d'une question.

Existence du problème. L'existence d'un problème de violence en milieu de travail peut entraîner des réactions chez certains employés qui adopteront des stratégies défensives au travail (Vacheret et Milton, 2007). L'existence du problème fut mesurée à partir de l'affirmation suivante: "L'intimidation du personnel est une problématique au sein des services correctionnels $d u$ Québec». Les points d'ancrage de cette échelle variaient entre un et quatre: «tout à fait d'accord»; «d'accord»; «en désaccord»; et «tout à fait en désaccord». En raison de la distribution des valeurs de la variable, cette échelle fut dichotomisée afin de distinguer ceux qui étaient d'accord ou tout à fait d'accord (valeur 1 ) de ceux qui étaient en désaccord ou tout à fait en désaccord (valeur 0 ).

Confiance des employés. La perte de confiance dans l'administration est synonyme de démoralisation des employés et d'insatisfaction au travail, elles-mêmes génératrices de comportements plus autoritaires vis-à-vis des détenus (Hepburn et Albonetti, 1980). La confiance des employés fut mesurée à partir de l'affirmation suivante: "J'ai confiance dans la capacité et la volonté d'agir de mon organisation si je dénonce des actes d'intimidation à mon égard.» Les points d'ancrage de cette échelle sont identiques à ceux de la première échelle. En raison de la distribution des valeurs de la variable, cette échelle fut dichotomisée afin de distinguer ceux qui étaient d'accord ou tout à fait d'accord (valeur 0 ) de ceux qui étaient en désaccord ou tout à fait en désaccord (valeur 1).

Tolérance. Confrontés à de l'intimidation répétitive, les gardiens peuvent en venir à être plus tolérants face à la violence. La tolérance fut mesurée à partir de l'affirmation suivante: «Un certain niveau d'intimidation est normal compte tenu de la nature de mon travail.» Les points d'ancrage de cette échelle sont identiques à ceux de la première échelle. En raison de la distribution des valeurs de la variable, cette échelle fut dichotomisée afin de distinguer ceux qui étaient d'accord ou tout à fait 
d'accord (valeur 1) de ceux qui étaient en désaccord ou tout à fait en désaccord (valeur 0).

Phénomène d'abdication. Par un effet d'usure, l'intimidation peut engendrer la passivité, un phénomène déjà constaté au sein des policiers victimes d'intimidation de la part des motards criminalisés (Gomez del Prado, 2004). L'abdication fut mesurée à partir de l'affirmation suivante: "Je sais que si je ferme les yeux sur certaines choses, je risque moins de vivre des actes d'intimidation.» Les points d'ancrage de cette échelle sont identiques à ceux de la première échelle. En raison de la distribution des valeurs de la variable, cette échelle fut dichotomisée afin de distinguer ceux qui étaient d'accord ou tout à fait d'accord (valeur 1) de ceux qui étaient en désaccord ou tout à fait en désaccord (valeur 0 ).

\section{Les variables contrôles}

Trois autres variables ont été considérées étant donné leur influence potentielle sur les pratiques professionnelles. Premièrement, nous avons considéré le sexe des répondants. La valeur 0 a été donnée aux répondants de sexe féminin et la valeur 1 a été donnée aux répondants de sexe masculin. Dans notre échantillon, 55,3\% des répondants sont des hommes.

Deuxièmement, le nombre d'années d'expérience 4 a été colligé. Cette variable continue variait de 1 à 29 ans et présentait une forte asymétrie positive $(M=9,5$, É.-T. $=6,79)$. Les valeurs de la variable ont été transformées en leur base logarithmique naturelle, ce qui a permis de normaliser la distribution. Ainsi, nous avons obtenu une moyenne logarithmique de 1,92 (É.-T. $=0,93$ ) et une moyenne géométrique de 6,89 années chez les participants $(2,72)$.

Troisièmement, étant donné que les pratiques et même l'ampleur de la problématique de l'intimidation peuvent varier d'une prison à une autre (Gaes et McGuire, 1985 ; Dilulio, 1987; Farmer, 1988; McCorkle et al., 1995; Huebner, 2003; Wolff et al., 2007), une dernière variable a été créée à cet effet. Cette variable nominale a permis d'identifier le lieu de travail du participant. Dans la présente étude, $25,8 \%$ des répondants travaillaient à Bordeaux, 10,6\% au palais de justice et $63,6 \%$ à RDP. Aux fins des analyses multivariées, cette variable a été transformée en variable discrète $(\mathrm{k}-1)$.

4. La variable «âge du répondant» a été écartée de nos analyses en raison d'un problème de colinéarité avec les années d'expérience. 


\section{Stratégie analytique}

Dans le cadre de cet article, trois stratégies d'analyse ont été préconisées. Premièrement, les analyses descriptives ont permis d'établir le niveau de victimisation directe et vicariante au cours de la carrière, les formes et les fréquences des intimidations vécues dans les douze derniers mois et la nature des pratiques professionnelles des gardiens de prison. Deuxièmement, des comparaisons de moyennes à l'aide du test de t ont été réalisées. Ces analyses ont permis de vérifier de manière générale si les gardiens ayant vécu de l'intimidation étaient plus susceptibles de modifier leurs pratiques professionnelles. Troisièmement, des analyses de régression logistique ont été effectuées afin de tenir compte de l'effet des variables contrôles (Hosmer et Lemeshow, 2000).

\section{Présentation des résultats}

\section{Niveaux de victimisation et pratiques professionnelles}

La distribution des résultats, présentée au tableau 1 , indique que les expériences vicariantes de victimisation sont plus fréquentes que les expériences directes de victimisation. La majorité des intimidations directes surviennent de manière occasionnelle $(35,1 \%)$ tandis que la majorité des intimidations vicariantes arrivent de manière courante $(37,9 \%)$. Toutefois, les moyennes indiquent que ces deux formes d'intimidation sont expérimentées de façon occasionnelle durant la carrière des surveillants.

TABLEA U 1

Niveaux estimés de victimisation directe et vicariante au cours de la carrière

\begin{tabular}{|l|c|c|c|c|}
\hline & \multicolumn{3}{|c|}{ Intimidation directe } & \multicolumn{2}{c|}{ Intimidation vicariante } \\
\hline & $\mathrm{n}$ & $\%$ & $\mathrm{n}$ & $\%$ \\
\hline Jamais (1) & 26 & 19,8 & 16 & 12,1 \\
\hline Rarement (2) & 20 & 15,3 & 13 & 9,8 \\
\hline Occasionnellement (3) & 46 & 35,1 & 43 & 32,6 \\
\hline Souvent (4) & 31 & 23,7 & 50 & 37,9 \\
\hline Très souvent (5) & 8 & 6,1 & 10 & 7,6 \\
\hline & $M=2,81, \hat{E}_{\text {. }}$-T. $=1,18$ & $M=3,19$, E. - T. $=1,11$ \\
\hline
\end{tabular}


Une deuxième série d'analyses descriptives a été réalisée pour mesurer les actes d'intimidation rapportés dans la dernière année. Les résultats sont présentés au tableau 2 . Sur les 132 répondants, $75 \%(\mathrm{n}=99)$ ont affirmé avoir été intimidés au moins une fois. Le tableau 2 présente les résultats pour l'ensemble des actes d'intimidation dans la dernière année. De manière générale, les agents correctionnels rapportent en moyenne 2,81 formes d'intimidation différentes à une fréquence de 55,42 actes par année. Les formes d'intimidation les plus fréquentes sont les menaces voilées et les insultes $(M=32,35)$ suivies des menaces de porter plainte $(M=6,97)$, des allégations tendancieuses $(M=4,82)$ et des menaces de mort et de blessures graves $(M=3,67)$.

\section{TA B LEA U 2}

Fréquence et prévalence des différentes formes d'intimidation vécues dans la dernière année

\begin{tabular}{|l|c|c|c|}
\hline & \multicolumn{1}{|c|}{$\boldsymbol{M}$} & \multicolumn{1}{c|}{.-T. } & Prévalence \\
\hline Menaces voilées, allusions imprécises, insultes & 32,35 & 65,97 & $69,5 \%$ \\
\hline Allégations tendancieuses, atteintes à la réputation & 4,82 & 12,95 & $30,5 \%$ \\
\hline Menaces de porter plainte ou de poursuite & 6,97 & 24,02 & $50 \%$ \\
\hline Menaces de mort, de blessures graves & 3,67 & 21,29 & $39,4 \%$ \\
\hline Se faire photographier, surveiller, suivre & 1,96 & 20,98 & $5,3 \%$ \\
\hline Se faire encercler & 0,15 & 0,49 & $10,6 \%$ \\
\hline Se faire lancer un objet & 2,2 & 12,1 & $22 \%$ \\
\hline Voies de fait simples & 0,32 & 0,74 & $19,2 \%$ \\
\hline Voies de fait graves & 0,03 & 0,17 & $3 \%$ \\
\hline Complot pour meurtre & 0,02 & 0,15 & $2,3 \%$ \\
\hline Tentative de meurtre & 0 & 0 & $0 \%$ \\
\hline Diversité des intimidations & 2,81 & 2,4 & - \\
\hline Fréquence des actes & 55,42 & 96,28 & - \\
\hline
\end{tabular}

Par ailleurs, la prévalence de chaque acte d'intimidation permet d'apprécier le pourcentage de répondants ayant vécu au moins une fois ce genre d'intimidation. Les menaces voilées ont été rapportées par $69,5 \%$ des répondants, les menaces de porter plainte par $50 \%$, les menaces de mort par 39,4\% et les allégations tendancieuses par 30,5\% d'entre eux. Les actes physiques d'intimidation sont rapportés par une proportion plus restreinte de gardiens de prison: $22 \%$ d'entre eux ont 
rapporté s'être fait lancer un objet tandis que 19,2\% ont été victimes de voies de fait simples.

Une troisième série d'analyses descriptives ont permis d'établir les pratiques professionnelles des surveillants (résultats non présentés ici). Sur les 132 répondants, 92,3\% estiment que l'intimidation des détenus est une problématique, $46,6 \%$ n'ont pas confiance dans la volonté et la capacité d'agir de leur direction, 70,5\% tolèrent une certaine forme de violence en raison de la nature de leur travail et $45,8 \%$ abdiquent leur pouvoir pour éviter les victimisations.

\section{Relations entre victimisations et pratiques professionnelles}

Une première série d'analyses a été effectuée pour apprécier les relations entre les formes de victimisation vécues par les surveillants et leurs pratiques professionnelles. Les résultats aux tests de moyennes sont présentés au tableau 3. Les surveillants qui considèrent l'intimidation comme un problème dans leur milieu de travail ont vécu plus d'expériences directes et indirectes de victimisation au cours de leur carrière. Les épisodes récents d'intimidation ne semblent pas avoir un impact sur la reconnaissance d'un problème en milieu correctionnel. En ce qui a trait à la confiance dans la capacité et la volonté d'agir de la direction pour régler le problème de l'intimidation, seules les expériences vicariantes d'intimidation ont un impact significatif sur le niveau de confiance. Ainsi, les surveillants qui expriment de la méfiance envers leur direction ont été plus témoins de collègues victimes d'intimidation. Finalement, la fréquence des intimidations récentes semble avoir une relation significative avec le phénomène d'abdication. Les gardiens qui abdiquent leur pouvoir sont ceux qui ont subi le plus d'actes d'intimidation au cours de la dernière année.

Une deuxième série d'analyses a été effectuée pour identifier les facteurs d'impact des pratiques professionnelles. Le tableau 4 présente les résultats aux modèles de régression logistique. La pratique professionnelle «Tolérance» n'est pas présentée dans ce tableau car le modèle s'est avéré non significatif (R-carré $=8,40 \%$; khi-deux $=9,33 ; \mathrm{dl}=6$; $p=0,239)$. Étant donné qu'une proportion importante de la fréquence des actes d'intimidation est composée de menaces voilées, d'insultes et d'allusions imprécises (près de $60 \%$ ), une variable de la fréquence des menaces voilées a été créée. Une transformation logarithmique a été appliquée en raison d'une forte asymétrie positive. Cette variable a été 


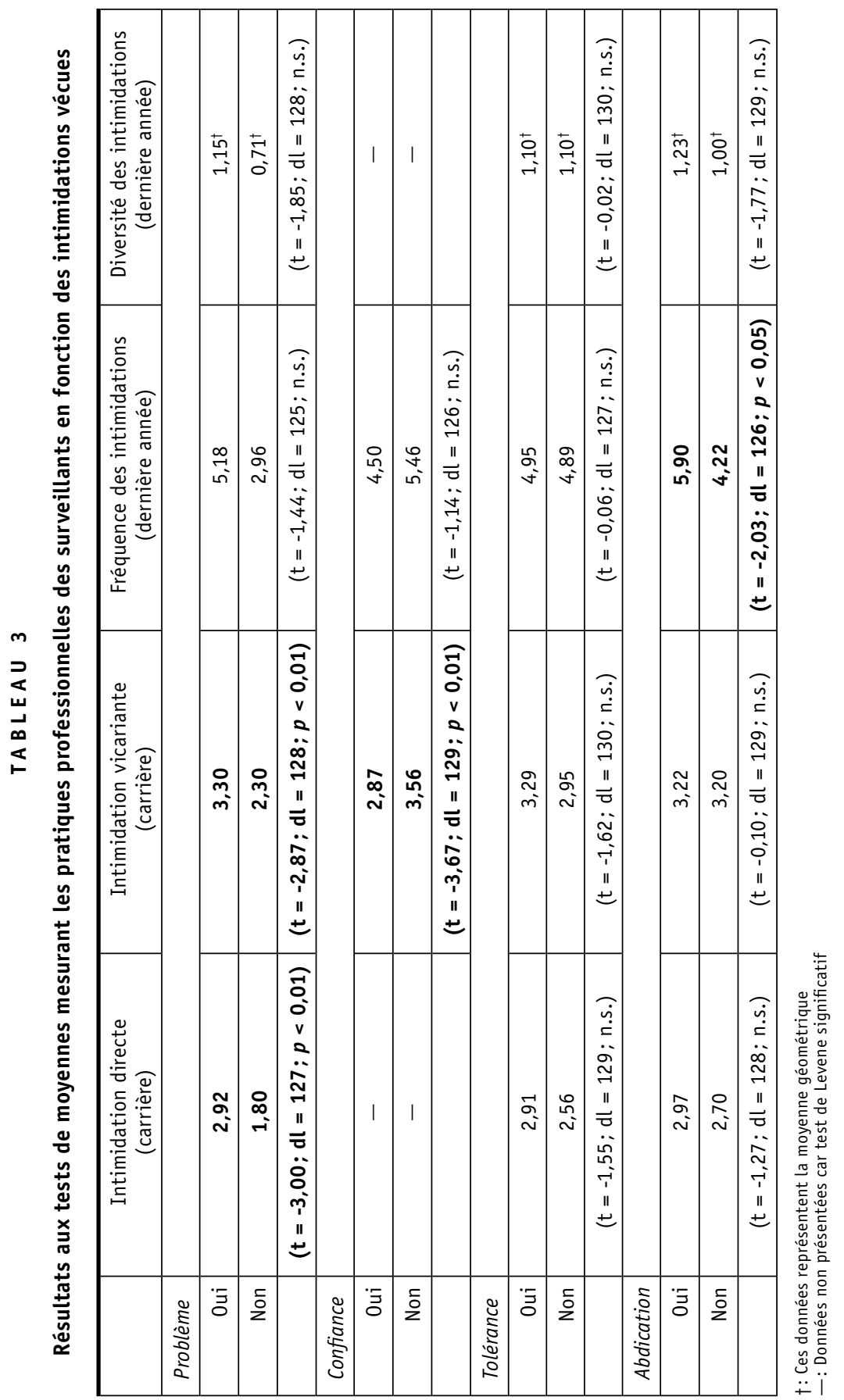




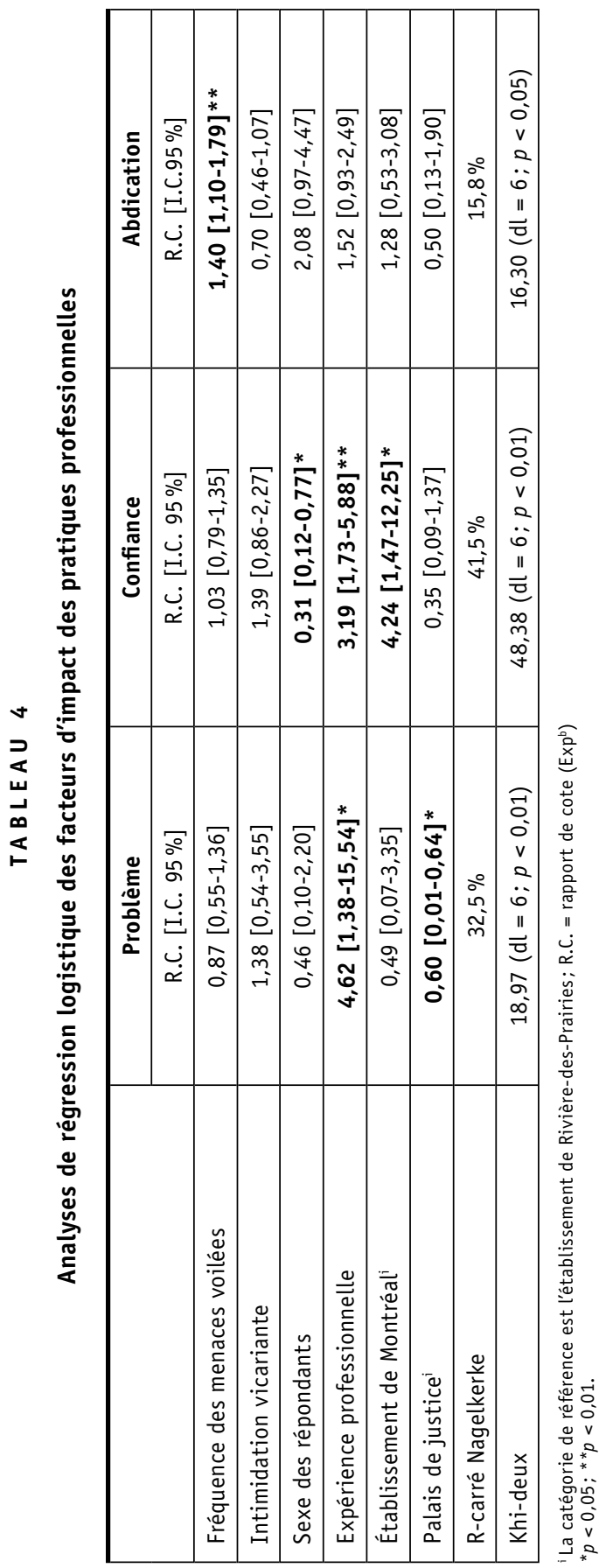


préférée à la variable mesurant la fréquence de l'ensemble des actes d'intimidation. Par ailleurs, la variable diversité des actes d'intimidation n'ayant pas engendré de résultats significatifs aux tests de moyenne, cette dernière n'a pas été intégrée aux analyses de régression logistique. Finalement, la variable de l'intimidation directe étant fortement corrélée avec la variable de l'intimidation vicariante, cette dernière variable a été privilégiée dans nos analyses.

Le premier modèle obtenu permet de prédire $32,5 \%$ de la reconnaissance d'un problème d'intimidation en milieu correctionnel. Ce modèle indique quaucune forme de victimisation ne semble avoir un impact sur la reconnaissance du problème. L'expérience professionnelle a un rapport de cote de 4,62 , ce qui indique que plus le surveillant gagne en expérience professionnelle, plus il a tendance à considérer que l'intimidation des détenus est un problème dans son milieu de travail. Le lieu de travail semble également avoir une influence sur la perception du problème. Les agents correctionnels travaillant au palais de justice sont moins susceptibles de considérer que l'intimidation des détenus est un enjeu dans leur milieu professionnel que leurs collègues de l'établissement de Rivière-des-Prairies (rapport de cote de 0,60).

Le deuxième modèle obtenu permet de prédire $41,5 \%$ du lien de confiance des agents envers leur administration. Ce modèle indique qu'aucune forme de victimisation ne semble avoir un impact sur le niveau de confiance. Par contre, le sexe des répondants a un rapport de cote de 0,31 indiquant que les femmes ont tendance à être plus méfiantes que les hommes. L'expérience professionnelle a un rapport de cote de 3,19, ce qui veut dire que plus l'agent correctionnel gagne en expérience professionnelle, moins il a confiance dans son organisation. Comme pour le modèle précédent, le milieu organisationnel semble également avoir un impact. Les gardiens travaillant à l'établissement de détention de Montréal sont beaucoup plus méfiants à l'égard de leur administration que leurs collègues de l'établissement de Rivière-desPrairies (rapport de cote de 4,24).

Le troisième modèle permet de prédire $15,8 \%$ de l'abdication du pouvoir des agents correctionnels. Avec un rapport de cote de 1,40, plus le surveillant subit des menaces voilées ou des insultes de la part de détenus plus il abdiquera son pouvoir. Les trois pratiques professionnelles analysées semblent donc être influencées par des facteurs différents, ce qui souligne qu'elles sont relativement indépendantes les unes des autres. 


\section{Interprétation des résultats}

De manière générale, nous constatons que les intimidations qui portent atteinte à l'intégrité physique sont relativement rares en milieu carcéral. Ainsi, les agressions physiques aggravées (voies de fait graves, complot pour meurtre) sont quasiment absentes de notre échantillon. Parallèlement, les agressions physiques mineures (voies de fait simples, se faire encercler ou se faire lancer un objet) sont peu fréquentes, mais leur prévalence indique que ces expériences de victimisation sont souvent communes au sein de la profession. Alors que les menaces verbales peuvent être proférées à l'endroit d'une personne en particulier, les agressions physiques les plus courantes en prison vont généralement faire plusieurs victimes. Lorsque le détenu se désorganise ou lorsque les risques inhérents à certaines tâches sont élevés, les surveillants vont normalement faire appel à d'autres collègues avant d'intervenir. Si une agression survient, elle risque alors de faire plusieurs victimes en même temps. Parallèlement, les intimidations verbales (insultes, menaces, fausses allégations) semblent ponctuer le quotidien des gardiens de prison si bien que les trois quarts des personnes interviewées ont rapporté au moins une forme d'intimidation dans la dernière année. À l'intérieur de cet échantillon, de $30 \%$ à $70 \%$ des répondants ont subi au moins une forme d'intimidation verbale de la part de détenus au cours de la dernière année. Ces résultats tendent à confirmer les conclusions des études portant sur les agressions des détenus envers les membres du personnel (Light, 1991; Jiang et Fisher-Giorlando, 2002; Useem et Piehl, 2006; Vacheret et Milton, 2007; Lahm, 2009; Sorensen et al., 2011).

Cette réalité se transpose également tout au long de la carrière du surveillant, ce dernier étant exposé à des intimidations directes ou vicariantes sur une base régulière. À long terme, ces expériences d'intimidation semblent saper le moral de l'ensemble des agents correctionnels. Qu'ils soient eux-mêmes intimidés par les détenus, qu'ils soient témoins de collègues victimes d'intimidation ou encore qu'ils en entendent parler, il en résulte une perte de confiance dans l'administration pénitentiaire. Nous assistons au décuplement des effets néfastes de la violence en milieu carcéral. À l'intérieur du microcosme de la prison, les surveillants développent une culture professionnelle relativement rigide et coupée, à la fois physiquement et symboliquement, de la société. Dans ces conditions, les expériences d'intimidation engendrent 
les mêmes répercussions, peu importe si elles sont vécues directement par le sujet ou si elles sont perçues à travers la victimisation des autres. Cette méfiance des surveillants à l'égard de leur hiérarchie peut se traduire par une importante insatisfaction au travail, elle-même génératrice de comportements plus sévères à l'égard des détenus (Hepburn et Albonetti, 1980).

Au-delà de cette perte de confiance généralisée, l'autre particularité de l'intimidation est d'engendrer une modification progressive des comportements des surveillants. Ce n'est pas tant l'acte en soi, mais l'effet cumulatif de plusieurs intimidations qui use le moral des troupes et transforme les pratiques professionnelles. Les formes d'intimidation les plus courantes en prison, les menaces voilées et les insultes, rythment le quotidien des surveillants à tel point que certains préfèrent fermer les yeux sur des infractions aux règlements ou des écarts de conduite pour éviter les victimisations. Si l'autorité des gardiens de prison se gagne par une application raisonnable et relative des règles en prison (Lombardo, 1981; Cooley, 1995; Sykes, 2007), leurs décisions ne peuvent faire l'objet de pressions indues.

Nos résultats mettent également en lumière des différences en fonction du lieu de travail des surveillants. Le niveau de confiance des employés de l'Établissement de Montréal est significativement plus bas que celui des employés de l'établissement de Rivière-des-Prairies, laissant ainsi présager des relations de travail problématiques entre les agents et leur direction. Comme nous le rappellent certains auteurs (Waddington et al., 2005; Parzefall et Salin, 2010), les politiques internes de gestion sont importantes dans l'interprétation de la violence en milieu de travail. Des procédures désuètes, des méthodes de travail mal adaptées à la réalité ou des directives mal comprises par le personnel peuvent contribuer à l'expérience de victimisation et à accroître la méfiance des employés envers leur direction. Ces éléments sont particulièrement importants dans la mise en place de moyens de prévention de la violence en milieu carcéral.

\section{Conclusion}

Alors que plusieurs études se sont penchées sur les répercussions des conflits de rôles et de la peur sur le travail des gardiens de prison, peu de recherches se sont intéressées à l'impact de leur victimisation. Par une approche exploratoire, nous avons pu identifier certains facteurs 
d'impact des pratiques professionnelles des agents correctionnels provinciaux. D'autres études sont toutefois nécessaires afin d'améliorer notre compréhension du phénomène de la violence psychologique perpétrée à l'égard des représentants du système judiciaire. Les pratiques professionnelles doivent notamment faire l'objet de mesures plus objectives. En nous limitant à des estimations individuelles du comportement des surveillants, notre étude a pu être influencée par le désir d'acceptabilité sociale au sein d'un milieu professionnel relativement hermétique. Des mesures telles que le nombre de rapports d'infraction rédigés par un surveillant, le nombre de plaintes formulées par un détenu ou encore le nombre de sanctions disciplinaires reçues par un surveillant, constituent des mesures objectives d'évaluation de la pratique professionnelle. Parallèlement, il convient de mesurer ces pratiques professionnelles à l'aide de plusieurs indicateurs différents afin de créer des échelles ayant des consistances internes satisfaisantes.

\section{Références}

Adler, J. R. (1998). Incidence of fear in prisons: Prisoner and officer assessments. Web Journal of Current Legal Issues, 5.

Agnew, R. (2002). Experienced, vicarious, and anticipated strain: An exploratory study on physical victimization and delinquency. Justice Quarterly, 19 (4), 603-632.

Ben-David, S., Silfen, P., \& Cohen, D. (1996). Fearful custodial or fearless personal relations: Prison guards' fear as a factor shaping staff-inmate relation prototype. International Journal of Offender Therapy and Comparative Criminology, 40 (2), 94-104.

Benguigui, G. (1997). Contrainte, négociation et don en prison. Sociologie $d u$ travail, 39 (1), 1-17.

Benguigui, G., Chauvenet, A., \& Orlic, F. (1992). Le personnel de surveillance des prisons. Essai de sociologie du travail. Droit et société, 22, 491-497.

Bittner, E. (1990). Aspects of police work. Boston: Northeastern University Press.

Chantraine, G. (2004). Par-delà les murs: expériences et trajectoires en maison d'arrêt. Paris: Presses Universitaires de France.

Cheek, F. E., \& Miller, M. D. S. (1983). The experience of stress for correction officers: A double-bind theory of correctional stress. Journal of Criminal Justice, 11 (2), 105-120.

Cooley, D. (1995). Social control and social order in male federal prisons (Doctoral thesis). University of Manitoba, Winnipeg.

Corrigan, R. S., Lester, D., \& Loftus, T. (1980). Perception of danger by police officers. Perceptual and Motor Skills, 50 (1), 284-284. 
Cusson, M., Gagnon, C., \& Gomez del Prado, G. (2011). L'intimidation envers les policiers du Québec (Résultats de recherche $n^{\circ} 3$ ). Montréal: Centre international de criminologie comparée.

DiIulio, J. J. (1987). Governing prisons: A comparative study of correctional management. New York: Free Press.

Farmer, J. F. (1988). A case study in regaining control of a violent state prison. Federal Probation, 52, 41-47.

Gaes, G. G., \& McGuire, W. J. (1985). Prison violence: The contribution of crowding versus other determinants of prison assault rates. Journal of Research in Crime and Delinquency, 22 (1), 41-65.

Gomez del Prado, G. (2004). L'intimidation exercée par les motards criminalisés sur les policiers du Québec Revue internationale de Criminologie et de Police technique et scientifique, 57 (2), 189-206.

Griffin, M. L. (2002). The influence of professional orientation on detention officers' attitudes toward the use of force. Criminal Justice and Behavior, 29 (3), 250-277.

Hepburn, J. R. (1985). The exercise of power in coercive organizations: A study of prison guards. Criminology, 23 (1), 145-164.

Hepburn, J. R., \& Albonetti, C. (1980). Role conflict in correctional institutions. Criminology, 17 (4), 445-459.

Hosmer, D. W., \& Lemeshow, S. (2000). Applied logistic regression (2 $2^{\text {nd }}$ ed.). New York: Wiley.

Huebner, B. M. (2003). Administrative determinants of inmate violence: A multilevel analysis. Journal of Criminal Justice, 31 (2), 107-117.

Jacobs, J. B., \& Retsky, H. G. (1975). Prison Guard. Journal of Contemporary Ethnography, 4 (1), 5-29.

Jiang, S., \& Fisher-Giorlando, M. (2002). Inmate misconduct: A test of the deprivation, importation, and situational models. The Prison Journal, 82 (3), 335-358.

Kauffman, K. (1988). Prison officers and their world. London: Harvard University Press.

Kratcoski, P. C. (1988). The Implications of research explaining prison violence and disruption. Federal Probation, 52, 27-32.

Lahm, K. F. (2009). Inmate assaults on prison staff: A multilevel examination of an overlooked form of prison violence. The Prison Journal, 89 (2), 131-150.

Light, S. C. (1991). Assaults on prison officers: Interactional themes. Justice Quarterly, $8(2), 243-261$.

Lin, W.-H., Cochran, J. K., \& Mieczkowski, T. (2011). Direct and vicarious violent victimization and juvenile delinquency: An application of general strain theory. Sociological Inquiry, 81 (2), 195-222.

Lombardo, L. X. (1981). Guards imprisoned: Correctional officers at work. New York: Elsevier

Lusignan, R. (1995). La victimisation des intervenants en criminologie: ses formes, son ampleur et ses conséquences sur la pratique clinique (Thèse de doctorat). Université de Montréal, Montréal. 
Lusignan, R. (1999). La victimisation des intervenants en criminologie: son ampleur et ses conséquences sur la pratique clinique. Forum, 11 (1), 35-39.

McCorkle, R. C., Miethe, T. D., \& Drass, K. A. (1995). The roots of prison violence: A test of the deprivation, management, and "Not-So-Total" institution models. Crime $\mathfrak{F}$ Delinquency, 41 (3), 317-331.

Nunnally, J. C., \& Bernstein, I. H. (1994). Psychometric theory (3 ${ }^{\text {rd }}$ ed.). New York: McGraw-Hill.

Ouimet, M. (1999). L'étonnante rareté de la violence contre le personnel dans les prisons. Forum, 11 (1), 25-29.

Parzefall, M.-R., \& Salin, D. M. (2010). Perceptions of and reactions to workplace bullying: A social exchange perspective. Human Relations, 63 (6), 761-780.

Patrick, S. (1998). Differences in inmate-inmate and inmate-staff altercations: Examples from a medium security prison. The Social Science Journal, 35 (2), 253-263.

Poole, E. D., \& Regoli, R. M. (1980). Role stress, custody orientation, and disciplinary actions. Criminology, 18 (2), 215-226.

Robinson, D., Lefaive, P., \& Muirhead, M. (1997). Sommaire des résultats du Sondage de 1996 auprès des employés du SCC (Rapport $\mathrm{n}^{\circ}$ B-17). Ottawa: Service correctionnel du Canada.

Rosine, L. (1992). Exposure to critical incidents: What are the effects on Canadian correctional officers? Forum, 4 (1).

Schaufeli, W. B., \& Peeters, M. C. W. (2000). Job stress and burnout among correctional officers: A literature review. International Journal of Stress Management, 7 (1), 19-48.

Seidman, B. T., \& Williams, S. M. (1999). L'impact des actes violents sur le personnel correctionnel. Forum, 11 (1), 30-34.

Sorensen, J. R., Cunningham, M. D., Vigen, M. P., \& Woods, S. O. (2011). Serious assaults on prison staff: A descriptive analysis. Journal of Criminal Justice, 39 (2), 143-150.

Stephan, J. J., \& Karberg, J. C. (2003). Census of state and federal correctional facilities, 2000. Washington, DC: Department of Justice, Bureau of Justice Statistics.

Sykes, G. M. (2007). The society of captives: A study of a maximum security prison. Princeton: Princeton University Press.

Triplett, R., Mullings, J. L., \& Scarborough, K. E. (1996). Work-related stress and coping among correctional officers: Implications from organizational literature. Journal of Criminal Justice, 24 (4), 291-308.

Useem, B., \& Piehl, A. M. (2006). Prison buildup and disorder. Punishment $\mathbb{E}$ Society, $8(1)$, 87-115.

Vacheret, M. (2001). L'univers des surveillants de prison: de la dévalorisation à l'atomisation. Revue de droit pénal et de criminologie, 532-559.

Vacheret, M., \& Lemire, G. (2007). Anatomie de la prison contemporaine (2 éd.). Montréal: Les Presses de l'Université de Montréal. 
Vacheret, M., \& Milton, M. (2007). Peurs en milieu carcéral: quand sentiments et expériences diffèrent. Criminologie, 40 (1), 185-211.

Waddington, P. A. J., Badger, D., \& Bull, R. (2005). Appraising the inclusive definition of workplace "violence". British Journal of Criminology, 45 (2), 141164.

Wolff, N., Blitz, C. L., Shi, J., Siegel, J., \& Bachman, R. (2007). Physical violence inside prisons: Rates of victimization. Criminal Justice and Behavior, 34 (5), 588-599.

ABSTRACT - Most of the studies have shown that serious inmate-on-staff assaults are relatively rare in prison. Rather, inmate-on-staff violence consists of minor physical assaults and psychological attacks. Although many studies have pointed out the factors that influence the professional practices of prison guards, very few have looked at the impact of violence on their work. We surveyed a sample of 132 provincial prison guards working in the Montreal area to establish the levels and the forms of intimidation they had experienced from inmates and to determine its impact on their professional practices. On the one hand, our results confirm the levels of inmate-on-staff violence found in previous studies. On the other, it appears that this violence changes some of their professional practices. Further studies are needed in order to validate the concept of professional practices of prison guards.

KEYWORDS - Workplace violence, criminal intimidation, prison guards, victimization, Professional practices.

RESUMEN - Las investigaciones sobre la victimización de los guardia-cárceles resaltan el carácter inusual de las agresiones severas hacia éstos de parte de los detenidos. Mas bien, el universo carcelario esta marcado por agresiones físicas menores y por ataques psicológicos contra los guardias. Si bien muchas investigaciones evidenciaron los factores de influencia de las prácticas profesionales de los guarda-cárceles, pocas se han explayado sobre el impacto de la violencia en su trabajo. Un cuestionario estructurado ha sido suministrado a 132 agentes correccionales que se desempeñan en prisiones de jurisdicción provincial dentro de la región de Montréal, con el propósito de establecer los niveles y las formas de intimidación vividas por dichos agentes, con el fin de evaluar los efectos de la victimización sobre su trabajo. Por un lado, nuestros análisis confirman las tendencias generales de la victimización de los guarda-cárceles. Por otro lado, dicha victimización pareciera modificar algunas prácticas profesionales. Sin embargo, más investigaciones son necesarias para validar el concepto de prácticas profesionales en el medio carcelario.

PALABRAS CLAVES - Violencia en el medio laboral, intimidación criminal, guardacárceles, victimización, práctica profesional. 